\title{
Self-field effects on flux flow in two-dimensional arrays of Nb Josephson junctions
}

\author{
E. Trías and T. P. Orlando \\ Department of Electrical Engineering and Computer Science, Massachusetts Institute of Technology, Cambridge, Massachsetts 02139
}

H. S. J. van der Zant

Department of Applied Physics, Delft University of Technology, Lorentzweg 1, 2628 CJ Delft, The Netherlands

(Received 14 March 1996)

\begin{abstract}
Measurements and numerical studies of the self-induced magnetic field effects on flux flow in twodimensional arrays of niobium Josephson junctions have been performed. It was found that the flux-flow resistance becomes larger as the penetration depth of the array decreases. A phenomenological model, which agrees qualitatively with the experiments and simulations, is presented to explain the self-field effects on flux flow. Due to the smaller spatial extent of supercurrents around a vortex when self-fields are important, both the mass of the vortex and the array viscosity decrease. The decreased mass and viscosity lead to an increase in flux-flow resistance. The effects of spin-wave damping are also discussed for underdamped arrays. Measurements and simulations on the spatial dependence of flux flow indicate that more complex dynamics is involved in the flux-flow regime than a simple linear flow of the vortices. [S0163-1829(96)02933-5]
\end{abstract}

\section{INTRODUCTION}

Two-dimensional arrays of Josephson junctions provide controllable model systems for the study of both vortex transport in superconductors and nonlinear dynamics of coupled systems ${ }^{1-3}$ as well as possible practical systems for terahertz oscillators. ${ }^{4,5}$ In this paper we will focus on the transport of vortices in discrete arrays which models the vortex motion in thin superconducting films.

An applied magnetic field will penetrate a twodimensional array in quantized bundles of flux known as vortices. The magnetic field and circulating currents around each vortex are confined to a characteristic distance $\lambda_{\perp}$, the array penetration depth. In the zero-voltage state vortices remain pinned in the periodic potential of the array. When the applied current is large enough to depin the vortices out of this potential barrier, the vortices move across the array, inducing a voltage. This depinning current will depend on the potential barrier due to the discrete Josephson junctions and on the commensurability of the induced vortex lattice with the intrinsic periodic potential. ${ }^{6-8}$

In this regime of flux flow that begins after depinning, the dynamics of the vortices is most similar to the motion of vortices in thin superconducting films. The flux-flow voltage is nearly linear with the applied current, and it is possible to measure and calculate a flux-flow resistance $R_{\mathrm{ff}}$. Previous studies have considered arrays where the penetration depth is much larger than the size of the arrays, ${ }^{9-11}$ which is similar to ultrathin films of superconductors only above the Kosterlitz-Thouless-Berezinskii transition temperature. In contrast, in this paper we are concerned with junctions made of niobium, where $\lambda_{\perp}$ is much smaller than the size of the array, so that self-field effects become important. This regime is similar to thin films of conventional and hightemperature type-II superconductors, where the analogous penetration depth is smaller than the size of the system and the resulting fields and currents are confined by self-field effects to the length scale of the penetration depth. More- over, in some of the niobium junctions, $\lambda_{\perp}$ can be of the order of the lattice spacing $p$, where self-field effects begin to probe the discrete nature of the array; this is a regime that is not accessible with thin films. We find that the flux-flow resistance increases as the penetration depth gets smaller and hence the effects of self-fields cannot be neglected when $\lambda_{\perp}$ is smaller than the array size. We also study the effects of temperature, damping, and magnetic field on $R_{\mathrm{ff}}$.

In the following section, we describe the samples and the experiments. The standard phenomenological model of vortex motion is extended to include the effects of self-fields in Sec. III. Section IV compares the measurements and simulations. The conclusions are summarized in Sec. V.

\section{EXPERIMENTAL DETAILS}

Several different two-dimensional arrays of Josephson junctions were fabricated and measured in order to investigate the flux-flow resistance in a wide parameter range of the Stewart-McCumber parameter $\beta_{c}$ and the perpendicular magnetic field penetration depth $\lambda_{\perp}$. All of the samples were fabricated using the $\mathrm{Nb}-\mathrm{Al}_{2} \mathrm{O}_{x}-\mathrm{Nb}$ trilayer process with varying critical current densities. None of the junctions were externally shunted. The arrays considered in this paper are square in the sense that every superconducting island not at the boundaries is connected to four others. At the top and bottom boundaries, superconducting islands are connected to current leads via normal resistors in order to achieve a more uniform current injection. The boundaries in parallel with the current injection are free to allow flux to penetrate.

The relevant parameters are shown in Table I. Arrays H1, $\mathrm{H} 2$, and $\mathrm{T}$ have $15 \times 15$ cells while sample $\mathrm{P}$ has $47 \times 47$ cells and sample H3 $7 \times 7$ cells. Samples in group H were fabricated at Hypres. ${ }^{12}$ Sample P was fabricated at IBM by a planarized all-refractory technology ${ }^{13}$ (PARTS) and sample T was fabricated at MIT Lincoln Laboratories by a selective $\mathrm{Nb}$ anodization process (SNAP). ${ }^{14}$ The lattice spacing $p$ for the arrays ranges from about 10 to $20 \mu \mathrm{m}$. 
TABLE I. Parameters for the measured samples. $R_{n}$ is the normal state resistance of the Josephson junction, $A_{J}$ is the area of the junction, and $C_{J}$ the capacitance. $L_{S}$ is the self-inductance of the loop, $p$ is the lattice spacing of the array, $\beta_{c}$ is Stewart-McCumber parameter, and $\lambda_{\perp}$ is the perpendicular magnetic field penetration depth.

\begin{tabular}{lccccc}
\hline \hline Parameter & $\mathrm{H} 1$ & $\mathrm{H} 2$ & $\mathrm{H} 3$ & $\mathrm{P}$ & $\mathrm{T}$ \\
\hline$R_{n}(\Omega)$ & 260 & 19 & 37.7 & 29.6 & 46.6 \\
$A_{J}\left(\mu \mathrm{m}^{2}\right)$ & 9 & 9 & 9 & 1 & 9 \\
$C_{J}(\mathrm{fF})$ & 300 & 300 & 300 & 67 & 440 \\
$\beta_{c}(0)$ & 450 & 33.3 & 64.3 & 10.2 & 120 \\
$\lambda_{\perp}(0)$ & 2.17 & 0.17 & 0.31 & 0.45 & 0.36 \\
$I_{c}(0)(\mu \mathrm{A})$ & 7.3 & 91.3 & 50.9 & 64.4 & 40.8 \\
$J_{c}\left(\mathrm{~A} / \mathrm{cm}^{2}\right)$ & 80 & 1100 & 570 & 7000 & 450 \\
$p(\mu \mathrm{m})$ & 16.5 & 16.5 & 16.5 & 9 & 18 \\
$L_{s}(\mathrm{pH})$ & 25.1 & 32.8 & 25.0 & 13.6 & 28.0 \\
Size $(\mathrm{cells})$ & $15 \times 15$ & $15 \times 15$ & $7 \times 7$ & $47 \times 47$ & $15 \times 15$ \\
\hline \hline
\end{tabular}

The measurements were performed in a ${ }^{4} \mathrm{He}$ probe. Inside the vacuum can there is a $\mu$-metal shield surrounding the sample as well as a small magnet that is used to apply a perpendicular magnetic field of up to $300 \mathrm{mG}$. To reduce high-frequency noise, the leads from the sample pass through $\pi$ filters before measurements are taken. The data were recorded on a computer with the use of digital voltmeters and digital lock-in amplifiers. All measurements were done using standard four-probe measurement techniques.

The junction normal state resistance $R_{n}$ is determined from the measured array resistance, $R_{n \text {, array }}$, by the relation $R_{n}=R_{n, \text { array }}\left(N_{x}+1\right) / N_{y}$, and is independent of temperature. $N_{y}$ is the number of array cells in the direction of current flow, while $N_{x}$ is the number of cells perpendicular to the injected current. The $I_{c} R_{n}$ product of a junction equals 1.9 $\mathrm{mV}$ at $T=0 \mathrm{~K}$. By calculating $I_{c}(0)$ we can determine the Josephson inductance $L_{J}(0)=\Phi_{0} / 2 \pi I_{c}(0)$ and the array penetration depth $\lambda_{\perp}(0)=L_{J}(0) / \mu_{0} p$. To estimate $\beta_{c}$, we first calculate the junction capacitance $C_{J}$ by measuring the Fiske steps of a one-dimensional array. ${ }^{15}$ From this we can calculate $\beta_{c}(0)=2 \pi I_{c}(0) R_{e}^{2} C_{J} / \Phi_{0}$. Here $R_{e}$ is the effective resistance of the junction which in general will depend on temperature and voltage, but will approach $R_{n}$ as the temperature approaches $T_{c}$. The experiments presented were performed at temperatures close to $T_{c}$ and we approximated $R_{e}=R_{n}$ in all the calculations.

Typical current-voltage, $I-V$, characteristics vs applied magnetic field are shown in Fig. 1 for the flux-flow region. The applied magnetic field $B_{0}$ is measured in units of the frustration $f=B_{0} p^{2} / \Phi_{0}$. All the properties of the array are periodic in $f$ with period $f=1$. The depinning current $I_{\text {dep }}$ indicates the onset of the flux-flow region. There is a transition part of the $I-V$ curve from the depinning current to a linear region. The flux-flow resistance $R_{\mathrm{ff}}$ is defined as the slope of this linear region which is shown by a straight line for each $I-V$. The inset shows that the assigned resistance values are linear in $f$ up to $f \approx 0.3$. For larger values of $f$ up to $f=0.5, R_{\mathrm{ff}}$ is no longer linear with $f$ due to the increased interaction between vortices. At large currents, the flux-flow region becomes unstable and the $I-V$ enters a row-switched

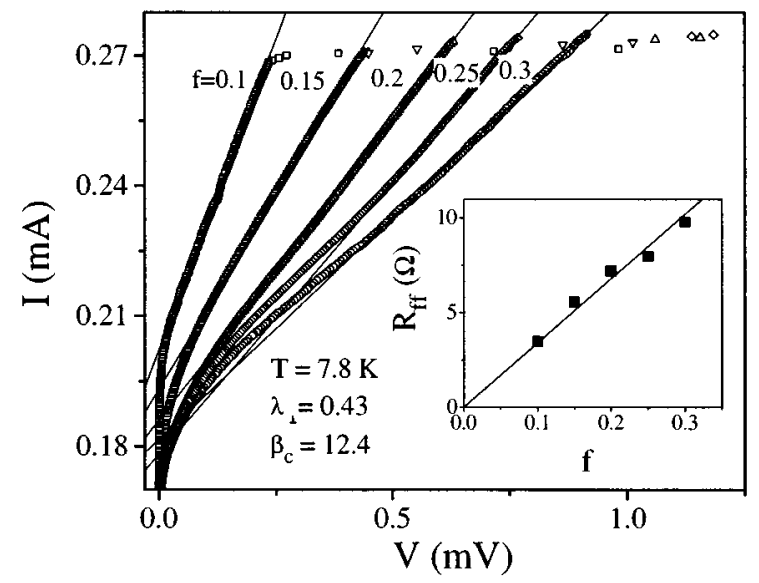

FIG. 1. Current-voltage characteristics for various magnetic fields from $f=0.1$ to 0.3 . The data is from sample $\mathrm{H} 2$ taken at $8.6 \mathrm{~K}$ with $\lambda_{\perp}=1.04$ and $\beta_{c}=5.1$. The numbers indicate values of $f$. The solid straight lines denote the linear region of flux flow. The inset shows that the flux-flow resistance $R_{\mathrm{ff}}$ is linear in $f$.

state which is not shown in Fig. 1.

It is also possible to measure the dynamic resistance $R_{d}$ directly by the use of a lock-in technique. A small ac excitation current is added to the dc bias current and the resulting voltage is measured with a lock-in amplifier. The ratio of the output voltage and input excitation currents results in $R_{d}$. Care was taken to use a frequency high enough to avoid the $1 / f$ noise of the amplifier while low enough to avoid cross talk in the lead wires. A frequency of $237 \mathrm{~Hz}$ was used for most of the lock-in measurements.

Figure 2 depicts a typical measurement of the $R_{d}$ vs dc bias current at different values of applied field. The regions of constant resistance in Fig. 2 are associated with flux flow and are to the right of the dotted line. This resistance is constant for frustrations that are less than 0.3. For higher values of $f$ the slope ceases to be linear and it is difficult to

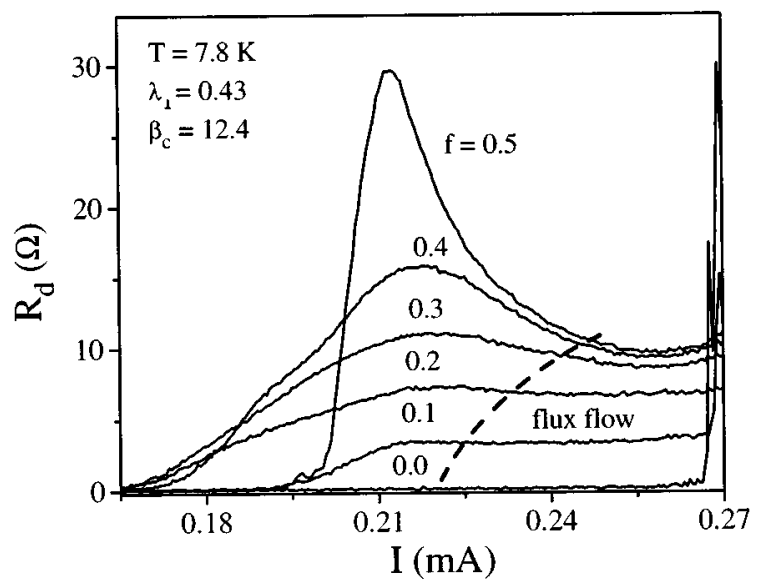

FIG. 2. The dynamic flux-flow resistance $R_{d}$ vs bias current measurements are taken by varying the magnetic field at $T=7.8 \mathrm{~K}$ for sample $\mathrm{H} 2$. The region to the right of the dashed line is the flux-flow region where $R_{d}$ is constant and proportional to $f$ for $f<0.3$ 


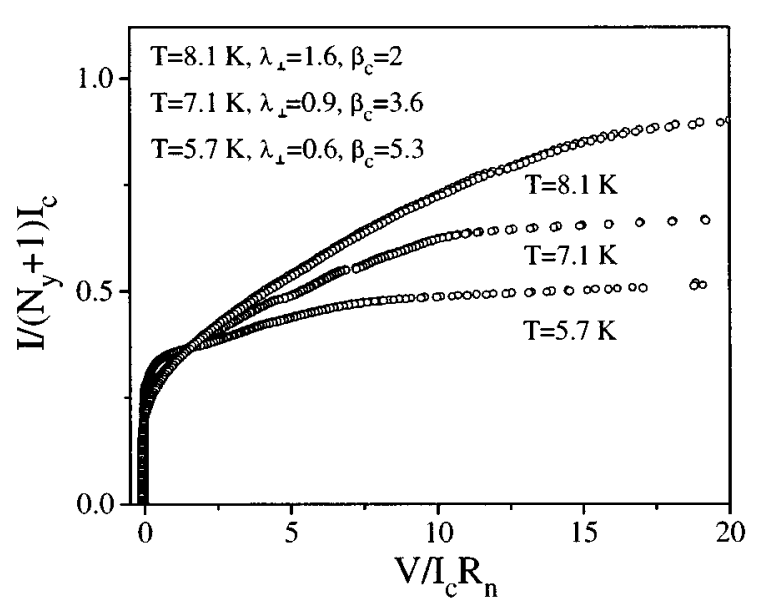

FIG. 3. Current-voltage characteristics taken by varying the temperature at $f=0.2$ for sample P. In this sample $\beta_{c}<5$ so that only the parameter $\lambda_{\perp}$ affects the slope. As $T$ increases, $\lambda_{\perp}$ decreases and $R_{\mathrm{ff}}$ increases.

assign an $R_{\mathrm{ff}}$. This nonlinearity may be due to the strong overlap and interaction of vortices in the high-frustration region.

As will be shown in later sections, before the linear region begins in the $I-V$, the applied current needs to be strong enough so that vortices overcome the intrinsic pinning force of the array lattice. For this reason $R_{\mathrm{ff}}$ is only well defined for a region well above $I_{\mathrm{dep}}$. Hence, the measured $R_{\mathrm{ff}}$ values are collected by performing a least-squares fit on the most linear part of the flux-flow region. The peak at $f=0.5$ is a consequence of the rising depinning current at the commensurate field. At $I=0.27$ there is a jump to the row-switched state. ${ }^{16,17}$

Figure 3 shows the temperature dependence of the fluxflow region. The junction critical current is assumed to follow the Ambegaokar-Baratoff ${ }^{18}$ dependence $I_{c}=0.86(\pi \Delta /$ $\left.2 e R_{n}\right) \tanh \left(\Delta / 2 k_{B} T\right)$, where $\Delta$ is the temperature-dependent quasiparticle excitation gap. Since the depinning current varies with temperature, both the measured current and voltage have been normalized by $\left(N_{x}+1\right) I_{c}$ and $I_{c} R_{n}$, respectively. This allows for a better comparison of the flux-flow slope. (Even when the applied current is normalized, the depinning currents will not be equal since they depend on the value of $\lambda_{\perp} \cdot{ }^{19,8}$ ) In this particular measurement the flux-flow resistance $R_{\mathrm{ff}}$ decreases with increasing temperature. For other arrays or temperature ranges, the flux-flow slope might have the opposite behavior with temperature. To better parametrize how the temperature affects $R_{\mathrm{ff}}$, we consider how temperature affects the parameters of the array.

By changing the temperature of the sample we can vary the $I_{c}$ of a junction, and hence change $\beta_{c}$ and $\lambda_{\perp}$ up to a factor of 5 in a controlled way. It is therefore possible to map out the $R_{\mathrm{ff}}$ dependence on both $\beta_{c}$ and $\lambda_{\perp}$. Recall that $\lambda_{\perp}$ is inversely proportional to $I_{c}$ while $\beta_{c}$ is proportional to $I_{c}$. Therefore, in the experiments $\lambda_{\perp}$ and $\beta_{c}$ cannot be varied independently and, as will be shown below, affect the fluxflow slope in opposite ways. To interpret the measurements more clearly we present a phenomenological model in Sec. III that explains the slope of the flux-flow region in terms of the junction parameters $\lambda_{\perp}$ and $\beta_{c}$ rather than explicitly on temperature.

In summary, our experiments show that there is a clear linear region in the $I$ - $V$ 's for $0<f<0.3$ which we characterize by a flux-flow resistance. The $R_{\mathrm{ff}}$ is linear in $f$, but depends on temperature through the parameters $\beta_{c}$ and $\lambda_{\perp}$.

\section{PHENOMENOLOGICAL MODEL}

In this section we present a phenomenological theory for the flux-flow resistance that explains the experimental dependence of the flux-flow resistance on the applied magnetic field, $\lambda_{\perp}$, and $\beta_{c}$. The effects of self-induced fields will also be analyzed. We will assume that vortices act as independent particles with a mass $M\left(\beta_{c}, \lambda_{\perp}\right)$ that experience a linear damping described by $\eta\left(\beta_{c}, \lambda_{\perp}\right)$. The array is taken to be infinite.

If the array is current biased in the $\hat{y}$ direction and the magnetic field is in the $\hat{z}$ direction the vortices will experience a Lorentz-like force in the $\hat{x}$ direction. The equation of motion of a vortex is

$$
M\left(\beta_{c}, \lambda_{\perp}\right) \ddot{x}+\eta\left(\beta_{c}, \lambda_{\perp}\right) \dot{x}=-\frac{d U(x)}{d x},
$$

where $U(x)$ includes the force of the driving current, $\Phi_{0} I_{\text {ext }} / p$, and the cell-to-cell potential barrier. ${ }^{6,19}$ The spatial properties of the barrier have been analyzed and for the $\lambda_{\perp}=\infty$ limit the potential due to the barrier can be schematically viewed as resembling an egg carton. This is the twodimensional version of the washboard potential of a Josephson junction. Since the vortices only travel in the $\hat{x}$ direction, the resulting slice of the "egg carton" potential is a cosine. The equivalent potential energy that describes the array is 11,20

$$
U(x)=-\frac{1}{2} \Delta E\left(\lambda_{\perp}\right) \cos \left(\frac{2 \pi x}{p}\right)-\frac{\Phi_{0}}{p} I_{\mathrm{ext}} x,
$$

where $\Delta E\left(\lambda_{\perp}\right)$ is the value of the energy barrier.

In overdamped systems $\left(\beta_{c}<1\right)$ with $\lambda_{\perp} \gg 1$ the mass ${ }^{21}$ has been found to be $M_{0}=\Phi_{0}{ }^{2} C / 2 p^{2}$, the energy barrier ${ }^{6}$ $\Delta E=0.2 E_{J}$, and the Bardeen-Stephen viscosity ${ }^{22}$ $\eta_{\mathrm{BS}}=\Phi_{0}^{2} / 2 p^{2} R_{n}$. This results in a flux-flow resistance $^{11,22,20}$ of $R_{\mathrm{ff}}=2 f R_{n \text {, array }}$. This resistance is a direct measure of the viscosity. For $\beta_{c}=0$ the viscosity is no longer linear and this model will break down. ${ }^{23}$ However, for $\beta_{c}>0$ the linear approximation is still accurate.

In the underdamped limit $\left(\beta_{c} \gg 1\right)$ and large $\lambda_{\perp}$, the Bardeen-Stephen damping is replaced by spin-wave damping ${ }^{24,20}$ with a viscosity $\eta_{\mathrm{sw}}=\Phi_{0}{ }^{2} / 4 \pi p^{2} \sqrt{L_{J} / C}$.

In this paper we are concerned with the effects of a finite $\lambda_{\perp}$ on $R_{\mathrm{ff}}$. It has been shown that $\Delta E$ increases as $\lambda_{\perp}$ gets smaller as ${ }^{19}$

$$
\Delta E=0.2 E_{J}\left(1+\frac{3}{2} \frac{1}{\lambda_{\perp}}\right)
$$

for $\lambda_{\perp}>1$. Following the quasistatic calculation of the vortex mass and Bardeen-Stephen viscosity as in Ref. 20, we find that both $M$ and $\eta_{\mathrm{BS}}$ decrease as $\lambda_{\perp}$ decreases. Specifically, we find 


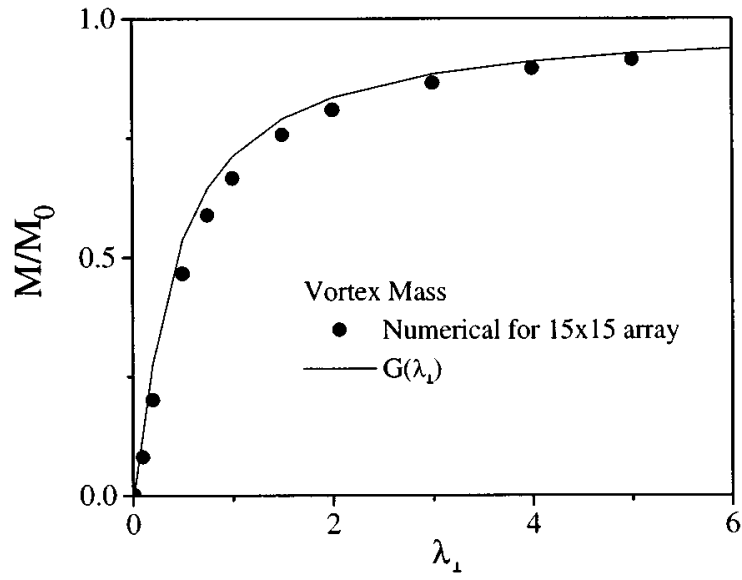

FIG. 4. Vortex mass normalized to the vortex mass at $\lambda_{\perp}=\infty$ calculated using the quasistatic approximations for a continuous film, $G\left(\lambda_{\perp}\right)$ (solid line), and numerically for a discrete Josephson array of $15 \times 15$ cells (solid dots).

$$
M=\frac{C \Phi_{0}^{2}}{2 p^{2}} G\left(\lambda_{\perp}\right)
$$

and

$$
\eta_{\mathrm{BS}}=\frac{\Phi_{0}^{2}}{2 p^{2}} \frac{1}{R_{n}} G\left(\lambda_{\perp}\right),
$$

where

$$
G\left(\lambda_{\perp}\right) \approx 1-\frac{1}{2} \sqrt{\frac{2}{\pi}} \frac{1}{\lambda_{\perp}}+\frac{7+3 \ln \left(8 \pi \lambda_{\perp}^{2}\right)}{48 \pi} \frac{1}{\lambda_{\perp}^{2}} .
$$

$G\left(\lambda_{\perp}\right)$ is plotted as a solid line in Fig. 4. The solid circles represent a quasistatic calculation for a $15 \times 15$ array where the sum of the junction voltages was calculated numerically taking into account all of the mutual inductances in the array. $M_{0}$ is the expected mass for $\lambda_{\perp}=\infty$. The quasistatic numerical calculation was also performed for arrays as small as $7 \times 7$ and as large as $31 \times 31$, and the results were almost identical as for the $15 \times 15$ array. This is not surprising since the mass is calculated by moving one vortex from one cell to the next and calculating the resulting voltage changes. Even if the size of the vortex, $\lambda_{\perp}$, is large, the largest voltage differences are found near the vortex core.

When $\lambda_{\perp}=\infty$ the vortex current falls off as $1 / r$ and the quasistatic calculation of the voltage involves the whole array. However, for finite $\lambda_{\perp}$, the current is $1 / r$ only out to about $\lambda_{\perp}$; hence a fewer number of junctions are involved in the calculation of the quasistatic voltages. Because $M \propto V^{2}$ and $\eta_{\mathrm{BS}} \propto V^{2}$, both the $M$ and $\eta_{\mathrm{BS}}$ will be smaller, for smaller $\lambda_{\perp}$. A detailed calculation of $G\left(\lambda_{\perp}\right)$ is given in the Appendix.

To combine all of the above concepts into a phenomenological picture of flux flow in a square array, we need to make one last simplifying assumption. We will postulate that the damping is separable into the Bardeen-Stephen component and the spin-wave damping as

$$
\eta=\eta_{\mathrm{sw}}+\eta_{\mathrm{BS}}
$$

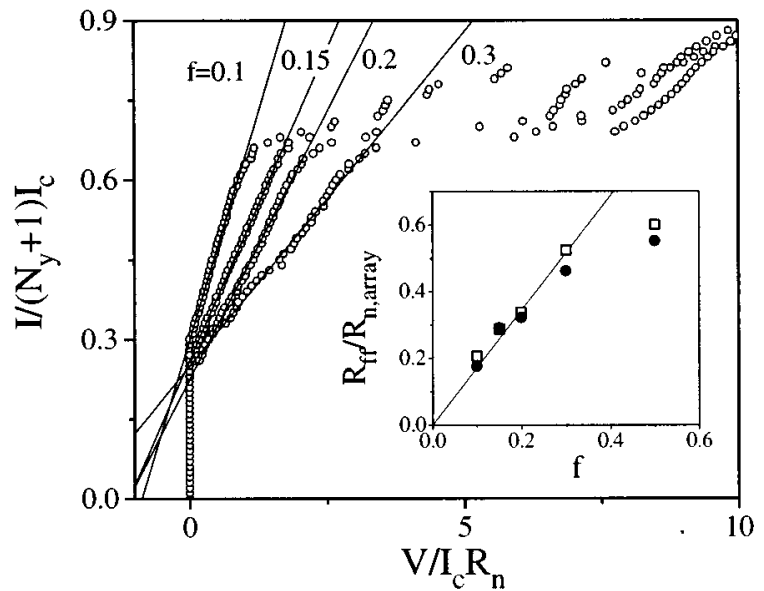

FIG. 5. Simulated $I$-V's for a $15 \times 15$ array with $\lambda_{\perp}=1$ and $\beta_{c}=6$ for various values of $f$. The solid straight lines denote the linear region of the flux flow. The inset shows $R_{\mathrm{ff}}$ vs $f$ for simulations (solid circles) and data on sample $\mathrm{H} 2$ (open squares). Both the data and simulations agree well and show that $R_{\mathrm{ff}}$ is linear in $f$ up to $f \approx 0.3$.

When $\beta_{c}$ is very small $\eta_{\mathrm{sw}}$ is almost negligible, and most of the losses will be due to ohmic dissipation. On the other hand, in the underdamped limit, $\eta_{\mathrm{sw}}$ will be substantially larger than $\eta_{\mathrm{BS}}$ and most of the losses will be due to spinwave damping. Using the calculated values of the appropriate damping it is possible to formulate the viscosity in terms of the array parameters

$$
\eta=\frac{\Phi_{0}^{2}}{2 p^{2}} \frac{1}{R_{n}} \frac{2 \pi G\left(\lambda_{\perp}\right)+\sqrt{\beta_{c}}}{2 \pi} .
$$

Again, since the junctions are effectively voltaged biased by the moving vortices, the viscosity is inversely proportional to the flux-flow resistance and

$$
R_{\mathrm{ff}}=2 f R_{n, \text { array }} \frac{2 \pi}{2 \pi G\left(\lambda_{\perp}\right)+\sqrt{\beta_{c}}} .
$$

With this result we now interpret the experimental data.

\section{COMPARISON WITH EXPERIMENTS AND SIMULATIONS}

To verify the validity of the phenomenological model and its range of applicability, a series of simulations and measurements was performed. Figure 5 shows numerical simulations of the $I-V$ 's. Since we are trying to characterize inductance effects, our simulations use a consistent set of Maxwell's equations and take into account the inductive interaction between all the cells of the array. Further details of the simulations are described elsewhere. ${ }^{25}$ The flux-flow regions of both the experimentally measured $I-V$ 's and the simulated $I-V$ 's are examined in the same manner as described in Sec. II. The flux-flow region is defined from the depinning until the voltage jumps to a row-switched state. As shown in the previous section, the array dynamics in the flux-flow state can be mostly described in terms of independent vortices. 


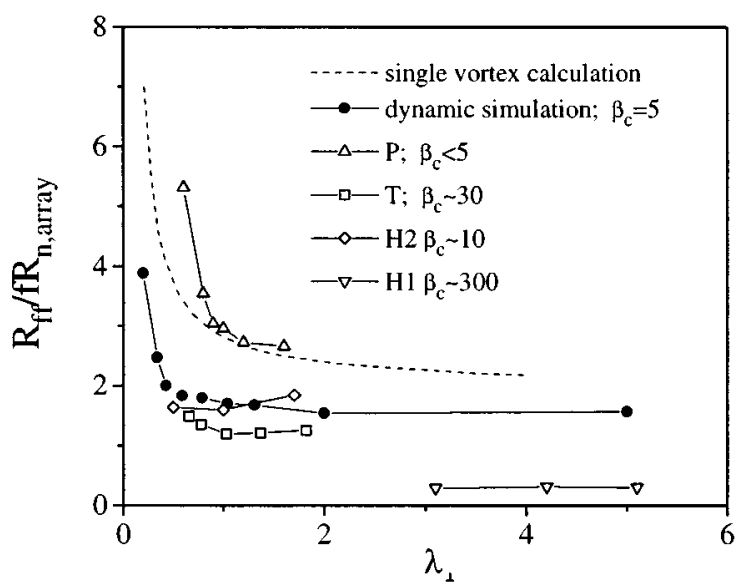

FIG. 6. Measured $R_{\mathrm{ff}}$ vs $\lambda_{\perp}$ for samples H1, H2, P, and T. The dashed line is a single-vortex approximation including only selffield effects [Eq. 5]. The solid circles are taken from dynamic simulations of a $15 \times 15$ array with $\beta_{c}=5$ and $f=0.2$. Note that $R_{\mathrm{ff}}$ is normalized to $f R_{n \text {,array }}$.

The inset of Fig. 5 shows a direct comparison of $R_{\mathrm{ff}}$ vs $f$ between the measured data (open squares) and the simulations (solid circles). The correspondence between simulation and experiment is very good for small values of $f$ and deviates only slightly for larger values. Also for values of $f<0.3$ the data and simulations have an approximate linear slope as predicted from the model.

Having shown that there is good agreement between the simulations and experiments in defining a flux-flow regime, we now focus on the dependence of $R_{\mathrm{ff}}$ on self-field effects as predicted by the phenomenological model. Figure 6 shows $R_{\mathrm{ff}}$ at $f=0.2$ for various samples and simulations as a function of $\lambda_{\perp}$. The smaller $\lambda_{\perp}$ is, the more self-fields effects are important. The simulations for $\beta_{c}=5$ and a $15 \times 15$ array are shown as open circles connected by a solid line. The prediction of the model, excluding spin-wave damping, is shown as the dotted line. The rest of the symbols mark the experimental data, with each symbol indicating a different sample. Different $\lambda_{\perp}$ values for the same sample are obtained by changing the temperature.

Although both the model and the simulations have a similar dependence on $\lambda_{\perp}$, the simulations lie below the model. This may be due to two reasons. First, the model assumes noninteracting vortices whereas the simulation includes vortex-vortex interactions. Second, we have found in simulations that larger arrays have higher $R_{\mathrm{ff}}$ than smaller arrays. It appears that for smaller arrays where edge effects are more pronounced the $R_{\mathrm{ff}}$ values are reduced. The simulations shown are for a $15 \times 15$ array whereas the model is for an infinite array.

The best experimental comparison with the model will be for overdamped arrays where spin-wave damping is small compared to Bardeen-Stephen damping. Sample $\mathrm{P}$ has $\beta_{c}<5$ and indeed shows the best trend. Note also that sample $\mathrm{P}$ is $47 \times 47$ and is larger than the size used in simulations. This may explain why the data lie above the simulations and closer to the model. Sample $\mathrm{H} 2$ has a moderate value of $\beta_{c} \sim 10$. The data on this $15 \times 15$ array lie closer to

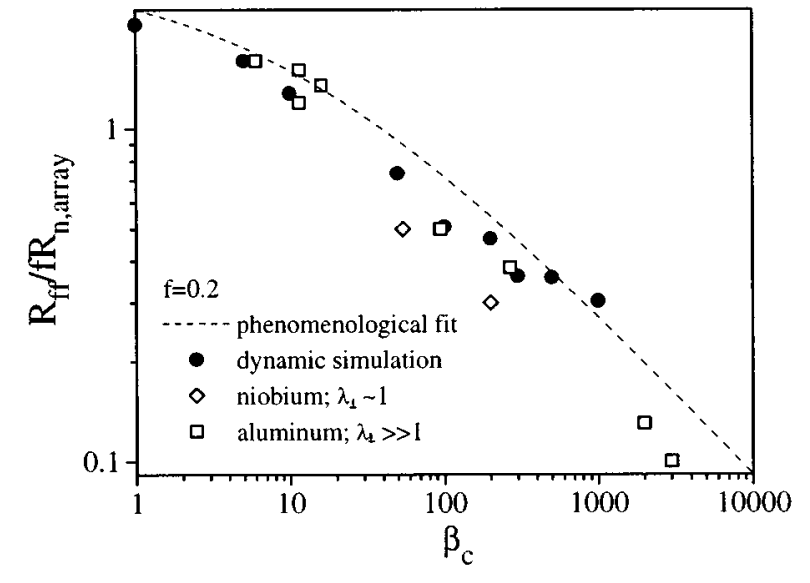

FIG. 7. Measured $R_{\mathrm{ff}}$ vs $\beta_{c}$ for samples $\mathrm{H} 1$ and a large aluminum array from Ref. 20. The solid circles are simulations with $\lambda_{\perp}=\infty$ for a $15 \times 15$ array with $f=0.2$. The dashed line is determined by adding the viscosities for Bardeen-Stephen flux flow and spin-wave damping.

the simulations and also show that $R_{\mathrm{ff}}$ increases as $\lambda_{\perp}$ decreases. Finally sample $\mathrm{H} 1$ has $\beta_{c}>300$ and spin-wave damping dominates so that in these samples the $\lambda_{\perp}$ dependence is obscured.

To characterize the $\lambda_{\perp}$ dependence more quantitatively it would be better to remove the $\beta_{c}$ effects from the measurements. We do this by first characterizing the $\beta_{c}$ dependence on $R_{\mathrm{ff}}$ when $\lambda_{\perp} \gg 1$ so that there are no self-field effects.

Figure 7 shows $R_{\mathrm{ff}}$ from both measurements on $\mathrm{Al}$ and $\mathrm{Nb}$ arrays, and numerical simulations. The $\mathrm{Al}$ measurements are from Ref. 20 and the arrays are $300 \times 100$ with $\lambda_{\perp} \gg 1$. The simulations are performed on a $15 \times 15$ array with $\lambda_{\perp}=\infty$. $\mathrm{The} \mathrm{Nb}$ data are measured from the $\mathrm{H}$ group of Table $\mathrm{I}$ and have a $\lambda_{\perp} \approx 1$. Both the data and simulations show that $R_{\mathrm{ff}}$ decreases as $\beta_{c}$ increases as expected. ${ }^{24}$ The dashed line represents the best fit of the simulation results to the phenomenological results but where we have generalized Eq. (9) to

$$
\frac{R_{\mathrm{ff}}}{f R_{N}}=\frac{2}{a+\left(\sqrt{\beta_{c}} / 2 \pi\right) b} .
$$

Equation 9 has $a=b=1$ for an infinite array. However, our simulations are necessarily for smaller arrays where edge effects might play a role. As stated above, the simulations show that smaller arrays have a slightly larger flux-flow resistance. Equation 10 is an effort to parametrize this effect for arrays of the same size as the data by fitting the simulations to a form given by the model. We find $a=2 / 3$ and $b$ $=4 / 3$ gives the best fit for the simulation results and is shown as the dashed line in Fig. 7.

We can now better characterize the $\lambda_{\perp}$ dependence of $R_{\mathrm{ff}}$. Figure 8 shows a normalized $R_{\mathrm{ff}}$ versus $\lambda_{\perp}$ where the $\beta_{c}$ dependence has been taken out by using Eq. (10). The dashed line represents the theoretical single-vortex calculation, while the solid circles are the dynamic simulations. The group of data for $\mathrm{H} 2$ has an improved fit to the theoretical prediction and the measured data for the $\mathrm{H} 1$ follow the simulations closely. In general, the trend of an increased flux-flow 


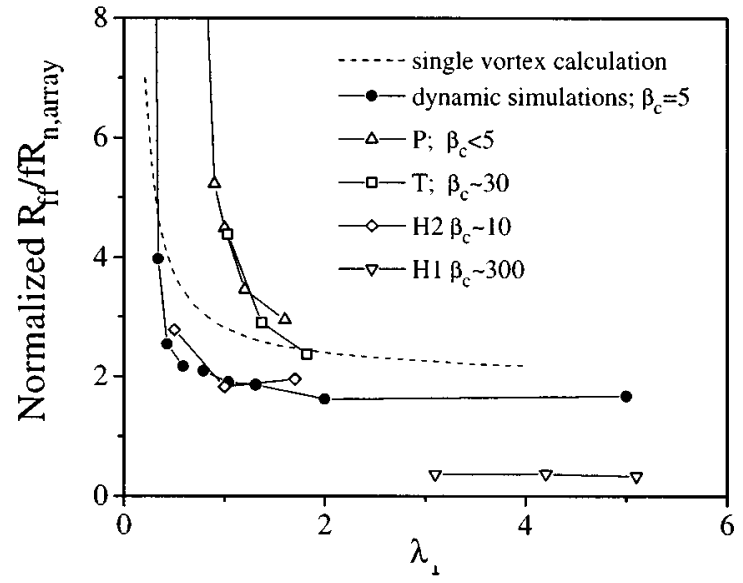

FIG. 8. Normalized $R_{\mathrm{ff}}$ vs $\lambda_{\perp}$. Samples H1, H2, P, and T are shown as open symbols. The dashed line is the single-vortex calculation of the model including only self-field effects [Eq. 5]. The solid circles are simulations on a $15 \times 15$ array for $\beta_{c}=5$.

slope as $\lambda_{\perp}$ decreases is more apparent in both the measurements and the simulations. Though the phenomenological theory does not explain all the aspects of the data, it does give a valid description of the $\lambda_{\perp}$ dependence of the viscosity of the array: strong self-induced fields reduce the viscosity of the array and increase $R_{\mathrm{ff}}$.

To further characterize these edge effects we fabricated sample H3 with voltage pads on every row. The simple phenomenological model presented assumes that the vortex motion is uniform over the array. However, data and simulations show that there is a spatial dependence for the vortex motion in our small array. We found that in sample H3 the first and last rows, the rows closest to the current injection and extraction, had very little flux flow. Most of the flow is through the center five rows. This was also observed in the simulations. Essentially, different rows have different fluxflow resistances. This spatial dependence is probably caused by the small size of the array. In general we find that in the flux-flow region of smaller arrays, some rows are mostly quiet and others have vortices flowing through them. This implies that there is interesting spatial dynamics and that flux flow cannot just be described by vortices moving incoherently through an array.

\section{CONCLUSIONS}

In the flux-flow regime the $I-V$ characteristics can be mostly explained in terms of a simple phenomenological model of vortex transport. These vortices represent bundles of magnetic flux that are driven by the applied current. Furthermore, vortices in the flux-flow region are localized and, because of the capacitive energy stored in the junctions, can be treated as massive particles. The applied field specifies the density of vortices present in the array. With this density, driving force, and viscosity, we have modeled the dependence of the flux-flow resistance in terms of the array parameters $\lambda_{\perp}$ and $\beta_{c}$ and applied magnetic field $f$. A linear dependence of $R_{\mathrm{ff}}$ vs $f$ is found.

It has also been shown that $R_{\mathrm{ff}}$ is dependent on both $\lambda_{\perp}$ and $\beta_{c}$. The dependence on $\beta_{c}$ follows from spin-wave damping while the $\lambda_{\perp}$ dependence is a result of a reduction of the viscosity caused by the decreasing physical size of vortices as $\lambda_{\perp}$ decreases. This simple phenomenological model gives a qualitative as well as a semiquantitative description of the dynamics. These effects have been corroborated in both experiments and in numerical simulations that take into account all the mutual inductances between cell pairs.

However, the flux-flow region appears to be richer in its dynamics than the presented model can account for. For small arrays, there is a spatial dependence of the flux-flow region which we have measured and also seen in simulations. Different rows have different flux-flow slopes and the outer rows closest to the edge appear to have almost no flux flow associated with them. Also, the effective linear damping as the sum of the Bardeen-Stephen damping and spin-wave damping is probably an oversimplification. Though these deviations do not diminish the useful and intuitive results from the phenomenological model, they do point the way for further research on the richness of the dynamics in the flux-flow regime.

\section{ACKNOWLEDGMENTS}

We thank Amy Duwel, Joel Phillips, Mauricio Barahona, Shinya Watanabe, and Steven Strogatz for valuable discussions. We are grateful to Jay Sage and Dave Feld at Lincoln Laboratories for their generous help. We thank Kevin Delin, Alan Kleisnasser, Ron Miller, and Mark Ketchen and acknowledge the support of AT\&T, IBM, and Lincoln Laboratories in the fabrication of the samples. We also acknowledge the support of the NSF under Grant No. DMR9402020. H.S.J.Z. was partly supported by the Netherlands Foundation for Fundamental Research on Matter (FOM).

\section{APPENDIX}

The effects of self-fields on the vortex mass and the viscosity are found by generalizing the quasistatic model of Ref. 22 to include the $\lambda_{\perp}$ dependence of the current. The electric energy $W_{E}$ stored in the capacitors gives rise to a vortex mass. Here

$$
W_{E}=\frac{1}{2} C \sum_{m, n} V_{m, n}^{2},
$$

where only nearest-neighbor capacitances, the geometric capacitance, have been used in the calculations. In this calculation the voltage is approximated from the static current distribution of a vortex moving with velocity $u$. Then $W_{E}=(1 / 2) M u^{2}$ and the proportionality constant $M$ can be viewed as a mass term of the kinetic energy.

As a first approximation, we assume that far from the vortex core we can linearize the basic Josephson current relation $J \approx J_{c} \phi$ where $d$ is the thickness of the film. If we let $u$ be the vortex velocity in $\hat{x}$ direction, then

$$
\frac{d \phi}{d t}=\frac{d}{d t} \frac{J}{J_{c}}=\frac{u}{J_{c}} \frac{\partial}{\partial x} J_{x}
$$

and similarly for the $\hat{y}$ direction. Therefore the resulting magnitude of the voltage, $V=\left(\Phi_{0} / 2 \pi\right)(d \phi / d t)$, is given by 


$$
|V|^{2}=\left(\frac{\Phi_{0}}{2 \pi} \frac{u}{J_{c}}\right)\left[\left(\frac{\partial J_{x}}{\partial x}\right)^{2}+\left(\frac{\partial J_{y}}{\partial x}\right)^{2}\right]
$$

From Eq. (A3), the square of the voltage is proportional to the square of the vortex velocity. Hence, the electrical energy of the array can be equated to the kinetic energy of the vortex

$$
\frac{1}{2} M u^{2}=\frac{1}{2} C \sum_{m, n} V_{m, n}^{2}
$$

where the sum is over all junctions in the array. In the continuum limit

$$
\sum V_{m, n}^{2} \approx \iint V^{2} \frac{d x}{p} \frac{d y}{p}
$$

and the resulting double integral after substituting Eq. (A3) is

$$
\sum V_{m, n}^{2} \approx\left(\frac{\Phi_{0}}{2 \pi} \frac{u}{J_{c} p}\right)^{2} \int_{\xi}^{\infty} \int_{\xi}^{\infty}\left[\left(\frac{\partial J_{x}}{\partial x}\right)^{2}+\left(\frac{\partial J_{y}}{\partial x}\right)^{2}\right] d x d y
$$

where $\xi$ represents the cutoff at the vortex core.

When the $\lambda_{\perp}$ is larger than the array size there are no induced fields and the vortex size is independent of the penetration depth. ${ }^{21}$ In this regime the "arctan" approximation can be used. The island phases can be estimated by $\theta=\arctan (y / x)$ and the circulating current $J=\left(I_{c} /\right.$ d) $\nabla \theta=\left(I_{c} / d r\right) \hat{\theta}$. This result has been used in Refs. 21 and 22 to find that $M_{0}=C \Phi_{0}^{2} / 2 p^{2}$.

If the penetration depth is smaller than the sample size, then the induced fields are important and the current distribution of a vortex is no longer described by the "arctan", expression. However, it can be approximated well by the equation due to Pearl $^{26}$ for the surface current density for a vortex in a thin film,

$$
J=\frac{I_{c}}{d} \frac{1}{r} \frac{1}{1+r / 2 \lambda_{\perp} p} \hat{\theta}
$$

Substituting $J_{x}$ and $J_{y}$ into Eq. (A6) and converting to cylindrical coordinates, we can estimate $\Sigma V^{2}$ by using the vortex core cutoff used by Eckern and $\operatorname{Schmid}^{21}$ of $p / \sqrt{2 \pi}$. We find that

$$
\sum V_{m, n}^{2} \approx 2\left(\frac{\Phi_{0} u}{2 p}\right)^{2} G\left(\lambda_{\perp}\right)
$$

where

$$
G\left(\lambda_{\perp}\right)=1-\frac{1}{2} \frac{1}{\lambda_{\perp}} \sqrt{\frac{2}{\pi}}+\frac{1}{8} \frac{\ln (\alpha \sqrt{2 \pi})}{\lambda_{\perp}^{2} \pi}+\frac{1}{4} \frac{1}{\pi \lambda_{\perp} \alpha}
$$

$$
+\frac{1}{4} \frac{1}{\pi \alpha^{2}}-\frac{1}{3} \frac{\lambda_{\perp}}{\pi \alpha^{3}}
$$

and

$$
\alpha=\frac{1}{2} \sqrt{\frac{2}{\pi}}+2 \lambda_{\perp} .
$$

This equation describes the effect on the sum of the junction voltages when the vortex size is decreased from the $\lambda_{\perp}=\infty$ case. The following equation approximates Eq. (A9) accurately:

$$
G\left(\lambda_{\perp}\right) \approx 1-\frac{1}{2} \sqrt{\frac{2}{\pi}} \frac{1}{\lambda_{\perp}}+\frac{7+3 \ln \left(8 \pi \lambda_{\perp}^{2}\right)}{48 \pi} \frac{1}{\lambda_{\perp}^{2}} .
$$

Here $\lambda_{\perp}$ is the usual normalized penetration depth.

Therefore, the mass equals

$$
M=\frac{C \Phi_{0}^{2}}{2 p^{2}} G\left(\lambda_{\perp}\right) .
$$

With a Bardeen-Stephen-like model for flux flow, we can equate the viscous energy dissipated by a vortex moving between adjacent cells to the power dissipated in each junction. ${ }^{20}$ If the vortex is moving at a constant velocity $u$, then the power dissipated by the viscous medium is equal to the power dissipated in all the junctions:

$$
\eta_{\mathrm{BS}} u^{2}=\sum_{m, n} V_{m, n}^{2} / R_{e}
$$

$R_{e}$ represents the effective voltage bias resistance of the junction which depends on temperature and voltage, and the sum is over all the junctions in the array. For most of the measurements, the temperature is near to $T_{c}$ and $R_{e} \approx R_{n}$. Using the same approximations as when calculating the mass, it possible to estimate the sum of the squared voltages and calculate the damping coefficient,

$$
\eta_{\mathrm{BS}}=\frac{\Phi_{0}^{2}}{2 p^{2}} \frac{1}{R_{n}} G\left(\lambda_{\perp}\right) .
$$

As expected from the voltage-biased condition, the larger the resistance, the lower the dissipated power. This result is calculated for a steady-state motion of a vortex so that it is independent of $\beta_{c}$. 
${ }^{1}$ Y. Braiman, A.S. Landsberg, and K. Wiesenfeld, Appl. Phys. Lett. 67, 1935 (1995).

${ }^{2}$ Shinya Watanabe and Steven H. Strogatz, Physica D 74, 197 (1994).

${ }^{3}$ P. Hadley, M.R. Beasley, and K. Wiesenfeld, Phys. Rev. B 38, 8712 (1988).

${ }^{4}$ S.P. Benz and C.J. Burroughs, Appl. Phys. Lett. 58, 2162 (1991).

${ }^{5}$ K. Wiesenfeld, S.P. Benz, and P.A.A. Booi, J. Appl. Phys. 76, 3835 (1994).

${ }^{6}$ C.J. Lobb, David W. Abraham, and M. Tinkham, Phys. Rev. B 27, 150 (1983).

${ }^{7}$ Thomas C. Halsey, Phys. Rev. B 31, 5728 (1985).

${ }^{8}$ E. Trías, J.R. Phillips, H.S.J. van der Zant, and T.P. Orlando, IEEE Trans. Appl. Supercond. 5, 2707 (1995).

${ }^{9}$ J.S. Chung, K.H. Lee, and D. Stroud, Phys. Rev. B 40, 6570 (1989).

${ }^{10}$ F. Falo, A.R. Bishop, and P.S. Lomdahl, Phys. Rev. B 41, 10983 (1990).

${ }^{11}$ M.S. Rzchowski, S.P. Benz, M. Tinkham, and C.J. Lobb, Phys. Rev. B 42, 2041 (1990).

${ }^{12}$ Hypres Inc., Elmsfort, NY 10523.

${ }^{13}$ M.B. Ketchen, D. Pearson, A.W. Kleinsasser, C.K. Hu, M. Smyth, J. Logan, K. Stawiasz, E. Baran, M. Jaso, T. Ross, K. Petrillo, M. Manny, S. Basavaiah, S. Brodsky, S.B. Kaplan, W.J.
Gallagher, and M. Bhushan, Appl. Phys. Lett. 59, 2609 (1991).

${ }^{14}$ M. Bhushan and E.M. Macedo, Appl. Phys. Lett. 58, 1323 (1991).

${ }^{15}$ H.S.J. van der Zant, R.A.M. Receveur, T.P. Orlando, and A.W. Kleinsasser, Appl. Phys. Lett. 65, 2102 (1994).

${ }^{16}$ H.S.J. van der Zant, C.J. Muller, L.J. Geerligs, C.J.P.M. Harmans, and J.E. Mooij, Phys. Rev. B 38, 5154 (1988).

${ }^{17}$ Wenbin Yu and D. Stroud, Phys. Rev. B 46, 14005 (1992).

${ }^{18}$ V. Ambagaokar and A. Baratoff, Phys. Rev. Lett. 10, 486 (1963).

${ }^{19}$ J.R. Phillips, H.S.J. van der Zant, and T.P. Orlando, Phys. Rev. B 47, 5219 (1993).

${ }^{20}$ H.S.J. van der Zant, F.C. Fritschy, T.P. Orlando, and J.E. Mooij, Phys. Rev. B 47, 295 (1993).

${ }^{21}$ Ulrich Eckern and Albert Schmid, Phys. Rev. B 39, 6441 (1989).

${ }^{22}$ T.P. Orlando, J.E. Mooij, and H.S.J. van der Zant, Phys. Rev. B 43, 10218 (1991).

${ }^{23}$ T.J. Hagenaars, P.H.E. Tiesinga, J.E. van Himbergen, and Jorge V. Jose, Phys. Rev. B 50, 1143 (1994).

${ }^{24}$ U. Geigenmüller, C.J. Lobb, and C.B. Whan, Phys. Rev. B 47, 348 (1993).

${ }^{25}$ J.R. Phillips, H.S.J. van der Zant, and T.P. Orlando, Phys. Rev. B 50, 9380 (1994).

${ }^{26}$ J. Pearl, Appl. Phys. Lett. 5, 65 (1964). 